\title{
Molecular bases of hearing loss in multi-systemic mitochondrial cytopathy
}

Fernando Scaglia, $M D^{1,2}$, Chang-Hung Hsu, MD ${ }^{3}$, Haeyoung Kwon, $P h D^{4}$, Ren-Kui Bai, MD, $P h D^{1}$, Cherng-Lih Perng, $M D^{3}$, Hua-Mei Chang, PhD ${ }^{5}, P u$ Dai, $M D^{6}$, E. O’Brian Smith, PhD ${ }^{7}$, David A. H. Whiteman, $M D^{8}$, Annette Feigenbaum, $M D^{9}$, Andrea Gropman, $M D^{10}$, and Lee-Jun C. Wong, PhD ${ }^{1}$

\begin{abstract}
Purpose: Hearing loss is a common clinical feature in classic mitochondrial syndromes. The purpose of this study was to evaluate the diverse molecular etiologies and natural history of hearing loss in multi-systemic mitochondrial cytopathies and the possible correlation between degree of hearing loss and neurological phenotype. Methods: In this retrospective study we evaluated the clinical features and molecular bases of hearing loss associated with multi-systemic mitochondrial cytopathy. Forty-five patients with sensorineural hearing loss and definite diagnosis of mitochondrial cytopathy according to the published diagnostic criteria were studied. Results: The sensorineural hearing loss was progressive and for the most part symmetrical with involvement of the higher frequencies. Both cochlear and retrocochlear involvement were found in this cohort. No correlation was found between the degree of hearing loss and the number and severity of neurological manifestations. Deleterious mtDNA point mutations of undisputed pathogenicity were identified in 18 patients. The A3243G mutation was the most frequently encountered among this group. MtDNA depletion, over-replication, and multiple deletions were found in further 11 cases.

Conclusion: This study reveals an expanding spectrum of mtDNA abnormalities associated with hearing loss. No correlation was found between the degrees of hearing loss and the severity of neurological manifestations. Genet
\end{abstract}

Med 2006:8(10):641-652.

Key Words: mtDNA mutation, deafness, maternal inheritance, maternally inherited hearing loss, mitochondrial syndrome

Hearing loss is the most common neurosensory disorder in humans $s^{1,2}$ with an incidence of approximately 1 in 1,000 children. ${ }^{1}$ Hereditary deafness is extremely genetically heterogeneous with more than 40 autosomal dominant (DFNA), 30 autosomal recessive (DNFB), and $6 \mathrm{X}$-linked (DFN) genes for

From the ${ }^{1}$ Department of Molecular and Human Genetics, Baylor College of Medicine, Houston, Texas; ${ }^{2}$ Department of Genetics, Texas Children's Hospital, Houston, Texas; ${ }^{3}$ Department of Neurology (C-HH) and Division of Clinical Pathology (C-LP), Tri-Service General Hospital, National Defense Medical Center, Taipei, Taiwan; ${ }^{4}$ Department of Applied Chemistry, Dong-duk Women's University, Seoul, Korea; ${ }^{5}$ Vita Genomics, Inc., Taipei, Taiwan; ${ }^{6}$ Department of Otolaryngology, 301 General Hospital, Beijing, People's Republic of China; ${ }^{7}$ Department of Pediatrics, Baylor College of Medicine, Houston, Texas; ${ }^{8}$ Shire Human Genetic Therapies, Inc., Cambridge, Massachusetts; ${ }^{9}$ Division of Clinical and Metabolic Diseases, The Hospital for Sick Children and University of Toronto, Toronto, Ontario, Canada; and the ${ }^{10}$ Department of Pediatrics, Georgetown University Medical Center, Washington DC, and Medical Genetics Branch, National Human Genome Research Institute, NIH, Bethesda, MD

Lee-Jun C. Wong, PhD, Department of Molecular and Human Genetics, Baylor College of Medicine, One Baylor Plaza, NAB2015, Houston, Texas 77030.

Supplementary Table 1 is available via the Article Plus feature at www.geneticsinmedicine. org. Please go to the October issue and click on the Article Plus link posted with the article in the Table of Contents to view this table.

Submitted for publication April 20, 2006.

Accepted for publication July 10, 2006.

FS and C-HH contributed equally to this article.

DOI: 10.1097/01.gim.0000237781.10594.d1 non-syndromic deafness that account for $60-70 \%$ of inherited hearing impairment. ${ }^{3}$ The most common cause for non-syndromic autosomal recessive hearing loss is caused by mutations in Connexin 26, a gap junction protein encoded by the GJB2 gene. ${ }^{4-6}$ However, about $30-40 \%$ of hereditary deafness is syndromic presenting with other clinical features in addition to hearing impairment. More than 30 nuclear genes, including those encoding transcription factors and gap junction proteins in Waardenburg syndrome and Usher syndrome, have been identified as responsible for syndromic hearing loss. ${ }^{3}$ Although the majority of cases with hereditary hearing loss are caused by nuclear gene defects, in recent years, it has become clear that mitochondrial genes and nuclear genes that affect mitochondrial biogenesis and function also play an important role.

Sensorineural hearing loss (SNHL) is one of the most prevalent and recognized clinical features of mitochondrial cytopathies. ${ }^{2,7}$ Mutations in mitochondrial DNA (mtDNA) can cause non-syndromic hearing loss. ${ }^{2,8,9}$ The most well-studied mutation is the (A1555G) mutation in the mitochondrial $12 \mathrm{~S}$ rRNA gene causing the non-syndromic hypersensitivity to ototoxic effects of aminoglycosides by increased binding of aminoglycosides to mitochondrial ribosomes, leading to the disruption of mitochondrial protein synthesis. ${ }^{10,11}$ Another recently identified mutation in the mitochondrial $12 \mathrm{~S}$ rRNA gene is the $\mathrm{T} 1494 \mathrm{C}$ in the conserved stem structure of $12 \mathrm{~S}$ 
rRNA. ${ }^{12}$ Other nucleotide changes at position 961 in the12S rRNA gene have been found to be associated with hearing loss. However, further studies are required to confirm whether and how often these changes would predispose carriers to aminoglycoside toxicity. ${ }^{13,14}$ Several mutations in the mitochondrial tRNA $^{\text {ser(AGY) }}$ gene are also known to cause maternally inherited non-syndromic hearing loss (MIHL) by disturbing the tRNA structure and function. ${ }^{15}$

Mitochondrial respiratory chain disorders are clinically and genetically heterogeneous, with central nervous system, skeletal, and cardiac muscle being the most susceptible tissues, and often manifest as multi-organ dysfunction that can include hearing loss as a clinical feature. The most well-known mtDNA mutations associated with multi-systemic disease and hearing loss are the A3243G mutation associated with MELAS (mitochondrial encephalopathy, lactic acidosis, and stroke-like episodes) syndrome and the large mtDNA deletion in KearnsSayre syndrome (KSS). The A3243G mutation and large mtDNA deletions have been found in large pedigrees of maternally inherited diabetes and deafness (MIDD). ${ }^{16,17}$ Other mtDNA mutations may also cause syndromic deafness. ${ }^{2}$

A systematic clinical and molecular analysis of patients with hearing loss and associated multi-systemic mitochondrial cytopathies has never been conducted using the established modified adult diagnostic criteria for the diagnosis of mitochondrial disease. These criteria have been adapted in order to improve the detection of mitochondrial cytopathies in children. ${ }^{18}$ The clinical aims of the study were to evaluate the natural history of hearing loss in these patients. We determined if there was a correlation between the degree of hearing loss and the observed neurological features in this group of patients. In addition, we report the results of an exhaustive investigation of the mtDNA defects in this cohort. A comprehensive clinical study and molecular analysis of mtDNA mutations in patients with hearing loss and associated multi-systemic mitochondrial cytopathies is relevant to facilitate genetic counseling and appropriate patient care.

\section{METHODS}

\section{Patients and specimens}

A retrospective study with review of medical records was conducted at Texas Children's Hospital, Georgetown University Medical Center, Children's National Medical Center, The Hospital for Sick Children in Toronto, and The Mayo Clinic to clinically evaluate patients with suspected mitochondrial disease between January, 1998 to December, 2003. Blood and/or skeletal muscle tissue samples from these patients were sent to the Molecular Genetics Laboratory, Institute for Molecular and Human Genetics, at Georgetown University Medical Center for molecular diagnosis of mtDNA disorders during that period of time. DNA was extracted from blood or skeletal muscle specimens according to published procedures. ${ }^{19,20}$ The clinical summaries, diagnostic studies, and demographic information of these patients were thoroughly reviewed concomitantly with the molecular analysis of submitted specimens. The study was approved by the Georgetown University Medical Center Internal Review Board and informed consent was obtained from the human subjects involved.

Clinical parameters that were evaluated in addition to hearing loss included developmental delay, abnormalities of muscular tone, movement disorder, seizures, stroke-like episodes, peripheral neuropathy, ophthalmologic disease, cardiomyopathy, arrhythmia, gastrointestinal symptoms, and endocrinopathy (including diabetes).

Relevant findings in diagnostic studies that were analyzed included central nervous system imaging abnormalities, lactic acidosis, histopathologic evidence of mitochondrial proliferation, cytochrome c oxidase deficiency by enzyme histochemistry, ultrastructural abnormalities of mitochondria, types of RC defect by biochemical analysis, and mtDNA mutations.

Patients were classified using the modified diagnostic criteria with definite, probable, and possible mitochondrial disease. ${ }^{18}$ Only the patients with definite diagnosis were included in this analysis. In this study, a systematic evaluation of mutations in nuclear genes encoding for mitochondrial respiratory chain protein subunits or involved in mtDNA biosynthesis was not performed.

\section{Audiological evaluation}

A complete audiological evaluation consisted of measurements of pure-tone and speech audiometry, tympanometry, acoustic reflexes, evoked and distortion-product otoacoustic emissions, as well as auditory brainstem evoked responses. However, not all patients had all these evaluations depending on the patient's age, developmental maturity, and level of cooperation.

\section{Analysis of common mtDNA mutations}

The samples were analyzed for the presence of large deletions and 11 common point mutations; A3243G, T3271C, G3460A, A8344G, T8356C, G8363A, T8993G, T8993C, G11778A, G14459A, and T14484C, by southern blot and allele specific oligonucleotide (ASO) dot blot analysis, respectively, according to published procedures. ${ }^{21,22}$

\section{Mutational analysis of mtDNA mutations responsible for mitochondrial non-syndromic hearing loss}

DNA samples from all patients with hearing loss were analyzed for the presence of mtDNA mutations responsible for mitochondrial non-syndromic hearing loss. Two primer pairs, $\mathrm{mtF} 1351 / \mathrm{mtR} 1762$ and $\mathrm{mtF} 7234 / \mathrm{mtR} 7921$ (the number stands for the $5^{\prime}$ nucleotide position of the 20-mer primer) were used in multiplex PCR/ASO analysis for point mutations: A1555G, T7445C, 7242insC, T7510C, T7511C, and T7512C. The PCR products were dotted onto nylon membrane followed by hybridization with wild type or mutant ASO probes as described previously. ${ }^{21,22}$ In addition, the T961G mutation in the mitochondrial 12S rRNA gene was analyzed by PCR/ RFLP method using forward primer mtF770; 5'CAATGCAGCTCAAAACGC3' and reverse primer mtR1156; 5'GGTCCTTTGAGTTTTAAGC3' for amplification followed by AciI 
(recognizes CCGC) restriction enzyme digestion that gives 2 fragments (127 and $275 \mathrm{bp}$ ) for wild type mtDNA and 3 fragments $(127,62$, and $213 \mathrm{bp})$ for mutant mtDNA with T961G mutation.

\section{Deletion analysis by PCR}

Multiple deletions not detectable by Southern analysis were investigated by PCR using mtF8295/mtR13738 and mtF8295/ mtR14499 primer pairs followed by agarose gel analysis. Deletions were also confirmed by real time quantitative PCR (real time qPCR) analysis (see below).

\section{Whole mitochondrial genome mutational analysis using temporal temperature gradient gel electrophoresis (TTGE)}

Mutations in the entire mitochondrial genome were studied by TTGE analysis using 32 pairs of overlapping primers according to published procedures. ${ }^{23}$ To identify the nucleotide sequence alterations, DNA fragments showing abnormal TTGE banding patterns were sequenced by direct DNA sequencing of the PCR product using a BigDye terminator cycle sequencing kit (Perkin Elmer) and an ABI 377 (Applied Biosystem) automated sequencer.

\section{Real-time quantitative PCR analysis of mtDNA content}

Southern undetectable multiple deletions and mtDNA depletion were analyzed simultaneously with real-time qPCR method. For mtDNA, two regions, np3212-3319 (tRNA ${ }^{\mathrm{Leu}(\mathrm{UUR})}$ and np12093-12170 (ND4) were amplified for the measurement of mtDNA content. Region np3212-3319 is almost always (>97\%) present in all mtDNA molecules including the deletion mutants. Therefore this region can be probed for the total mtDNA amount. Region np12093-12170 is deleted in $97 \%$ of the deletion molecules that have been reported. Thus, probe in this region is used to measure the amount of nondeletion molecules. The difference in the amount of total and nondeletion mtDNA molecules is the amount of deletion mtDNA molecules. The beta- 2 microglobulin (b2M) gene is used as the nuclear gene (nDNA) normalizer for the calculation of mtDNA to nDNA ratio. The target sequences were detected by using TaqMan probes; 6FAM-5'TTACCGGGCTCTGCCATCT3'-TAMRA, 6FAM-5'CATCATTACCGGGTTTTCCTCTTGTA3'-TAMRA, and VIC-5'TTGCTCCACAGG TAGCTCTAGGAGG3'-TAMRA for mt DNA regions np32123319 and np12093-12170, and $2 M$ respectively. The primers are: 5'CACCCAAGAACAGGGTTTGT3' (forward) and 5'TGGCCATGGGTATGTTGTTA3' (reverse) for mtDNA np32123319 region; 5'TCCTCCTATCCCTCAACCCC3' (forward) and 5'CACAATCTGATGTTTTGGTTAAAC3' (reverse) for mtDNA np12093-12170 region; and 5'TGCTGTCTCCATGTTTGATGTATCT3' (forward) and 5'TCTCTGCTCCCCACCTCTAAGT3' (reverse) for b2M gene.

The $20 \mu \mathrm{L}$ PCR reaction contained $1 \times$ Platinum qPCR SuperMix-UDG Master Mix (Invitogen, Carsbad, CA), $300 \mu \mathrm{M}$ of each primer, $100 \mathrm{nM}$ of TaqMan probe, $0.4 \mu \mathrm{L}$ of Rox dye (supplied by the manufacturer), and $2 \mu \mathrm{g}$ of total genomic DNA extract. Real-time PCR conditions were 2 minutes at $50^{\circ} \mathrm{C}, 10$ minutes at $95^{\circ} \mathrm{C}$, followed by 40 cycles of 15 seconds of denaturation at $95^{\circ} \mathrm{C}$, and 60 seconds of annealing/extension at $60^{\circ} \mathrm{C}$. Real-time $\mathrm{qPCR}$ analysis was performed on Sequence Detector System ABI-Prism 7700. ${ }^{24-26}$ The cut-off of $<30 \%$ and $>140 \%$ of age-matched mean mtDNA content to be significant for mtDNA depletion and over-amplification was arrived based on our previous studies of approximately 350 muscle specimens. ${ }^{24-26}$

\section{Mutational analysis of GJB2 gene}

The entire coding region of GJB2 gene was amplified by PCR using forward primer F131; 5'TCTGTCCTAGCTATGTTCCT3', and reverse primer R926; 5'ATCCCTCTCATGCTGTCTAT3', followed by direct DNA sequencing using BigDye terminator cycle sequencing kit (Perkin Elmer) and an ABI 377 (Applied Biosystem) automated sequencer. The sequencing results were compared with the wild type GJB2 sequence in the GenBank (accession number M86849).

\section{Statistical analysis}

Statistical analysis was performed using SPSS (SPSS Inc, Chicago, IL). Kruskal Wallis was used to compare medians. Regression analysis was used to calculate correlation coefficient.

\section{RESULTS}

\section{Patients}

One thousand five hundred subjects suspected of mitochondrial disorders were evaluated for mitochondrial cytopathies in the different centers participating in the study. Among them, one hundred fifteen patients were determined to have a definite diagnosis of mitochondrial disease according to the modified diagnostic criteria (Table 1). ${ }^{18}$ Of these 115, 45 (40\%) were found to have sensorineural hearing loss (SNHL) in addition to other features of mitochondrial disease. Among these 45 patients, $30(66 \%)$ had a complete audiological evaluation. Mutations in connexin 26 (GJB2) gene were not detected in our patients. The age range of our subjects was between 5 months and 83 years of age with a median age at onset of hearing loss of 7 years. Overall, the SNHL in this cohort was symmetric and progressive. Seventy percent (31/45) of the patients had cochlear involvement, 15\% (7/45) had retrocochlear involvement, and 15\% (7/45) had both. The gender ratio in our cohort (0.73), exhibited a slight female predominance that was not statistically significant $(P=0.23)$.

The predominant clinical presentations were classical mitochondrial syndromes in 16 patients and non-specific encephalomyopathy in 15 patients. No correlation was found between the degree of severity of hearing loss and the number and severity of neurological symptoms (hypo- or hypertonia, ataxia, movement disorder, ptosis, external ophthalmoplegia, frequency of seizures and stroke-like episodes, peripheral neuropathy, brainstem alterations, visual impairment, and mental retardation). 
Table 1

Modified diagnostic criteria applied to subjects who were referred for evaluation of mitochondrial disease $\left({ }^{\circledR} \mathrm{MDBU} ; \mathrm{N}=1,500\right)^{a}$

\begin{tabular}{|c|c|c|}
\hline & Major criteria & Minor criteria \\
\hline Clinical & $\begin{array}{l}\text { Clinically complete RC encephalomyopathy }{ }^{b} \text { or } \\
\text { a mitochondrial cytopathy defined as } \\
\text { fulfilling all three of the following }\end{array}$ & Symptoms compatible with a RC defect ${ }^{d}$ \\
\hline Histology & $2 \%$ ragged red fibers (RRF) in skeletal muscle & $\begin{array}{l}\text { Smaller numbers or RRF, SSAM, or } \\
\text { widespread electron microscopy } \\
\text { abnormalities of mitochondria }\end{array}$ \\
\hline Enzymology & $\begin{array}{l}\text { Cytochrome c oxidase negative fibers or } \\
\text { residual activity of a RC complex }<20 \% \text { in a } \\
\text { tissue; }<30 \% \text { in a cell line, or }<30 \% \text { in } 2 \text { or } \\
\text { more tissues }\end{array}$ & $\begin{array}{l}\text { Antibody-based demonstration of an } \\
\text { RC defect or residual activity of an } \\
\text { RC complex } 20 \%-30 \% \text { in a tissue, } \\
30 \%-40 \% \text { in a cell line, or } 30 \%-40 \% \\
\text { in } 2 \text { or more tissues }\end{array}$ \\
\hline Functional & $\begin{array}{l}\text { Fibroblast ATP synthesis rates } 3 \text { SD below } \\
\text { mean }\end{array}$ & $\begin{array}{l}\text { Fibroblast ATP synthesis } 2-3 \text { SD below } \\
\text { mean, or fibroblasts unable to grow in } \\
\text { galactose media }\end{array}$ \\
\hline Molecular & $\begin{array}{l}\text { Nuclear or mtDNA mutation of undisputed } \\
\text { pathogenicity }\end{array}$ & $\begin{array}{l}\text { Nuclear or mtDNA mutation of } \\
\text { probable pathogenicity }\end{array}$ \\
\hline Metabolic & & $\begin{array}{l}\text { One or more metabolic indicators of } \\
\text { impaired metabolic function }\end{array}$ \\
\hline
\end{tabular}

ATP, adenosine triphosphate; SSAM, subsarcolemmal accumulation of mitochondria.

${ }^{a}$ This classification scheme was applied to 1,500 patients who were referred for evaluation of mitochondrial disease. The details of these criteria are described by Bernier et al. ${ }^{18}$

${ }^{b}$ Leigh disease, Alpers disease, Lethal infantile mitochondrial disease, Pearson syndrome, Kearns Sayre syndrome, MELAS, MERRF, NARP, MNGIE, and LHON.

${ }^{c} 1$ ) Unexplained combination of multi-systemic symptoms that is essentially pathognomonic for a RC disorder; 2) a progressive clinical course with episodes of exacerbation or a family history strongly indicative of a mtDNA mutation; 3 ) other possible metabolic or non-metabolic disorders have been excluded by appropriate testing.

${ }^{d}$ Added pediatric features: stillbirth associated with a paucity of intrauterine movement, neonatal death or collapse, movement disorder, severe failure to thrive, neonatal hypotonia, and neonatal hypertonia as minor clinical criteria.

\section{Categories of molecular defects in hearing loss associated with multi-systemic mitochondrial cytopathies}

All patients were analyzed for mtDNA common point mutations and deletions. If negative, they were subsequently subjected to TTGE analysis of the entire mitochondrial genome. If muscle specimens were available, they were also analyzed by real time quantitative PCR to determine mtDNA depletion and over-replication. Overall, 29/45 (64\%) patients with hearing loss associated with multi-systemic mitochondrial cytopathy harbored molecular defects in mtDNA (Table 2). Based on these findings, the patients were classified in three categories: I) patients with point mutations or single mtDNA deletions of undisputed pathogenicity; II) patients with multiple mtDNA deletions, and alterations in mtDNA copy number (depletion and over-replication); and III) patients with definite mitochondrial disease and mtDNA alterations of unknown significance (Table 3). The median age of onset of hearing loss in the three groups was 21 years, 3.5 years, and 3.5 years respectively $(P<0.001$ Kruskal Wallis).

Overall, 69\% (29/45) of the patients with hearing loss associated with multi-systemic mitochondrial cytopathy had identifiable pathogenic mtDNA alterations, including 14 subjects with pathogenic mutations in mitochondrial tRNA genes, 1 subject with a mutation in the mitochondrial $12 \mathrm{~S}$ rRNA gene, 1 subject with a mutation in a protein coding gene, 2 subjects with large deletions in mitochondrial DNA, and 11 subjects

\section{Table 2}

Summary of mtDNA alterations in patients with SNHL and multi-systemic mitochondrial disease

\begin{tabular}{lc}
\hline MtDNA alterations & Number of patients \\
\hline Undisputed mtDNA point mutations & 18 \\
A1555G & 1 \\
A3243G & 12 \\
A8344G & 1 \\
G14459A & 1 \\
G5783A tRNA ${ }^{\text {cys }}$ (heteroplasmy) & 1 \\
Single deletion & 2 \\
Multiple deletions, depletion, and over-replication & 11 \\
Depletion (30\% of age-matched mean) & 5 \\
Over-replication & 4 \\
Multiple deletion (20\% mutant load) & 1 \\
mtDNA depletion and multiple deletion & 1 \\
\hline
\end{tabular}

with alterations in mtDNA integrity and copy number (including multiple deletions, depletion, and/or over-replication). One of the patients affected with mtDNA depletion also harbored the mild primary LHON mutation G15257A and a secondary LHON mutation G15812A. Most of the patients had 
Table 3

Clinical and laboratory findings of patients with SNHL and multi-systemic mitochondrial disease ${ }^{a}$

\begin{tabular}{|c|c|c|c|}
\hline Number & Sex & $\begin{array}{c}\text { Onset } \\
\text { age of SNHL (y) }\end{array}$ & Mutation \% heteroplasn \\
\hline \multicolumn{4}{|c|}{$\begin{array}{l}\text { Group I: Patients with mtDNA point } \\
\text { mutations and single deletions } \\
\text { of undisputed pathogenicity }\end{array}$} \\
\hline $1 b$ & $\mathrm{~F}$ & 83 & $\begin{array}{l}\text { A1555G, 100\% } \\
\text { Multiple dln by PCR }\end{array}$ \\
\hline $2 \mathrm{~b}$ & M & 48 & A $3243 \mathrm{G}, 10 \%$ \\
\hline $3 b$ & $\mathrm{~F}$ & 43 & A3243G, $10 \%$ \\
\hline $4 \mathrm{~b}$ & $\mathrm{~F}$ & 9 & A3243G, 30\% \\
\hline $5 b$ & M & 15 & A3243G, $36 \%$ \\
\hline $6 \mathrm{~b}$ & $\mathrm{~F}$ & 22 & A3243G, $40 \%$ \\
\hline $7 \mathrm{~b}$ & $\mathrm{~F}$ & 18 & A3243G, $43 \%$ \\
\hline $8 \mathrm{bm}$ & M & 20 & $\begin{array}{l}\text { A3243G, } 42 \% \text { b, } 75 \% \mathrm{~m} \\
200 \% \text { mtDNA prolif }\end{array}$ \\
\hline $9 \mathrm{bhcm}$ & M & 16 & $\begin{array}{l}\text { A3243G, } 47 \% \text { b, } 72 \% \text { h, } \\
\quad 65 \% \text { c, } 78 \% \mathrm{~m}\end{array}$ \\
\hline $10 \mathrm{~b}$ & $\mathrm{~F}$ & 5 & A3243G, 66\%b \\
\hline $11 \mathrm{~b}$ & $\mathrm{~F}$ & 8 & A3243G, $57 \%$ b \\
\hline $12 \mathrm{~b}$ & $\mathrm{~F}$ & 25 & A3243G, $21 \%$ \\
\hline $13 b$ & $\mathrm{~F}$ & 35 & A $3243 \mathrm{G}, 5 \% \mathrm{c}, 4 \% \mathrm{~b}$ \\
\hline $14 \mathrm{~m}$ & M & 54 & A8344G, 95\% \\
\hline $15 \mathrm{~m}$ & $\mathrm{~F}$ & 25 & $3 \mathrm{~kb}$ deletion $(47 \%)$ \\
\hline $16 \mathrm{~m}$ & M & 30 & $\begin{array}{l}5 \mathrm{~kb} \text { common deletion } \\
(33 \%)\end{array}$ \\
\hline $17 \mathrm{~m}$ & $\mathrm{~F}$ & 2 & G14459A 100\% \\
\hline $18 \mathrm{~km}$ & $\mathrm{~F}$ & 1 & G5783A, 95\%k, 96\%m \\
\hline
\end{tabular}

Clinical, metabolic, histochemical, and biochemical features ${ }^{b}$ in addition to hearing loss

Hypotonia, ataxia, fatigue, muscle weakness, peripheral neuropathy, diabetes, migraine

Cardiomyopathy, diabetes

Stroke-like episodes, sz, muscle weakness, ragged red fibers

Sz, cardiomyopathy, lactic acidosis

Dd, stroke-like episodes, sz, failure to thrive, abnl brain MRI

Stroke-like episodes, muscle weakness, cardiomyopathy, ophthalmoparesis

Sz, dd, stroke-like episodes, short stature, cardiomyopathy, pancreatitis, GI dysmotility, lactic acidosis, abnl brain MRI

Dd, sz, stroke-like episodes, short stature, lactic acidosis

Dd, short stature, hypotonia, sz, pigmentary retinopathy, dilated cardiomyopathy, lactic acidosis, stroke-like episodes, abnl brain MRI

Short stature, failure to thrive, hypotonia, exercise intolerance

Short stature, hypotonia, exercise intolerance

Bipolar disorder, hypotonia, exercise intolerance

Bipolar disorder, cardiomyopathy and stroke like episodes

Ataxia, myoclonus, exercise intolerance, fatigue, muscle weakness, abnl brain MRI, renal tubular disease, lactic acidosis

Short stature, fatigue, muscle weakness, $\mathrm{rp}$, ptosis, ophthalmoplegia, bradycardia, COX negative fibers, enlarged and abnormal mitochondria on EM

CPEO, ptosis, dysarthria, proximal myopathy

Stroke, short stature, lactic acidosis, high CSF lactate, increased T2 signal in putamen, increased lactate in basal ganglia on brain MRS

Renal failure, peripheral neuropathy, exercise intolerance, fatigue, cardiomyopathy, short stature, cyclic vomiting, lactic acidosis, elevated CK, retinitis pigmentosa, FTT, RRF on light microscopy, abundant mitochondria with concentric cristae and electron dense inclusions on EM, CI, CIII, and CIV deficiency

Dd, hypotonia, proximal muscle weakness, lactic acidosis cardiomyopathy, exercise intolerance, elevated CK, variation of fiber size, vacuoles, COX deficient fibers, lipid accumulation and ragged red fibers on muscle histology, mitochondrial enlargement and abnormal cristae on EM

Migraine, fatigue, muscle cramps, bipolar disorder, rp, ophthalmoparesis, optic atrophy, abnl EMG, CI deficiency 
Table 3

Continued

\begin{tabular}{|c|c|c|c|c|}
\hline Number & Sex & $\begin{array}{c}\text { Onset } \\
\text { age of SNHL (y) }\end{array}$ & Mutation \% heteroplasmy & $\begin{array}{c}\text { Clinical, metabolic, histochemical, and biochemical features }{ }^{b} \\
\text { in addition to hearing loss }\end{array}$ \\
\hline $21 \mathrm{~m}$ & M & 28 & Dpln, $10 \%$ & $\begin{array}{l}\text { Dd, seizures, hypotonia, ataxia, muscle weakness, lactic } \\
\text { acidosis, ophthalmoparesis, CPEO, exocrine pancreatic } \\
\text { insufficiency, gonadal failure, diabetes, abnl brain MRI }\end{array}$ \\
\hline $22 \mathrm{~m}$ & $\mathrm{~F}$ & 24 & Over-replication $160 \%$ & $\begin{array}{l}\text { Muscle weakness, ptosis, visual loss, migraine, short stature, } \\
\text { ataxia, CPEO, type } 1 \text { fiber predominance, subsarcolemmal } \\
\text { mitochondrial accumulation }\end{array}$ \\
\hline $23 \mathrm{~m}$ & M & 3.5 & Over-replication $200 \%$ & $\begin{array}{l}\text { Dd, hypotonia, exercise intolerance, fatigue, muscle } \\
\text { weakness, failure to thrive, hepatic failure, low plasma } \\
\text { carnitine, elevated CK, fiber type variation, elongated } \\
\text { mitochondria, abnormal cristae, CII and CIV deficiency }\end{array}$ \\
\hline $24 \mathrm{~m}$ & M & 2 & Multiple dln, 57\% & $\begin{array}{l}\text { Hypotonia, ataxia, seizures, myoclonus, ptosis, CII } \\
\text { deficiency }\end{array}$ \\
\hline $25 \mathrm{~m}$ & M & 15 & $\begin{array}{l}\text { Multiple dln, 52\%, dpln, } \\
\quad 30 \%\end{array}$ & $\begin{array}{l}\text { Short stature, ptosis, headaches, stroke-like episodes, muscle } \\
\text { wasting, dysphagia }\end{array}$ \\
\hline $26 \mathrm{~m}$ & M & 0.1 & dpln, $30 \%$ & Dd, hypotonia, CI, CIII, and CIV deficiency \\
\hline $27 \mathrm{~m}$ & M & 1.5 & Dpln, $30 \%$ & $\begin{array}{l}\text { Dd, hypotonia, seizures, weakness, fatigue, exercise } \\
\text { intolerance, GI dysmotility, cyclic vomiting, lactic } \\
\text { acidosis, elevated CK, hypothyroidism, cardiomyopathy, } \\
\text { CIV deficiency }\end{array}$ \\
\hline $28 \mathrm{~m}$ & $\mathrm{~F}$ & 13 & $\begin{array}{l}\text { Dpln, 25\%, G15257A } \\
\text { (D171N) G15812A } \\
\text { (V356M) T4216C } \\
\text { (Y304H) }\end{array}$ & $\begin{array}{l}\text { Migraine, ataxia, peripheral neuropathy, fatigue, cerebellar } \\
\text { atrophy, COX negative fibers, increased lipid and } \\
\text { glycogen, enlarged mitochondria on EM, CI and CIII } \\
\text { deficiency }\end{array}$ \\
\hline $29 \mathrm{~m}$ & $\mathrm{~F}$ & 1 & $150 \%$ over-replication & Dd, hypotonia, GI dysmotility, lactic acidosis, CIII deficiency \\
\hline \multicolumn{5}{|c|}{$\begin{array}{l}\text { Group III: Patients with definite } \\
\text { mitochondrial disease and } \\
\text { mtDNA alterations of unknown } \\
\text { significance }\end{array}$} \\
\hline $30 \mathrm{~m}$ & $\mathrm{~F}$ & 1 & Multiple dln by PCR & Dd, sz, abnl brain MRI, GI dysmotility \\
\hline $31 \mathrm{~m}$ & M & 7 & Multi dln [sim] 15\% & $\begin{array}{l}\text { Dd, hypotonia, autistic features, sz, dementia, lactic acidosis, } \\
\text { myoclonus }\end{array}$ \\
\hline \multirow[t]{2}{*}{$32 \mathrm{~m}$} & $\mathrm{~F}$ & 2.5 & Multiple dln, by PCR & Dd, sz, muscle weakness, short stature \\
\hline & & & Dpln $70 \%$ & \\
\hline $33 b$ & $\mathrm{~F}$ & 1.5 & $\begin{array}{l}\text { 961delTins } \mathrm{C}_{3} \\
\quad \mathrm{C}_{5} \mathrm{TC}_{4} \ddot{\mathrm{y}} 1 \mathrm{AC}_{12}\end{array}$ & $\begin{array}{l}\text { Dd, sz, hypotonia, ophthalmoplegia, abnl brain MRI, lactic } \\
\text { acidosis, COX negative fibers, abnormal EM, CI deficiency }\end{array}$ \\
\hline $34 \mathrm{~b}$ & M & 6 & T961G & $\begin{array}{l}\text { Dd, hypotonia, headache, ataxia, sz, muscle weakness, GI } \\
\text { dysmotility, ragged red fibers, increased subsarcolemmal } \\
\text { mitochondrial accumulation }\end{array}$ \\
\hline $35 \mathrm{~m}$ & $\mathrm{~F}$ & 1.5 & Dpln, $85 \%$ & Dd, sz, lactic acidosis, CIV deficiency \\
\hline $36 \mathrm{~b}$ & M & 7 & U.S. & $\begin{array}{l}\text { Dd, encephalopathy, sz, myoclonus, heart block, GI } \\
\text { dysmotility, lactic acidosis, failure to thrive, microcephaly, } \\
\text { ragged red fiber, CI, CIII and CIV deficiency }\end{array}$ \\
\hline $37 \mathrm{~b}$ & $\mathrm{~F}$ & 6.5 & A1438G & $\begin{array}{l}\text { Cardiomyopathy, elevated transaminases, abnormal cristae } \\
\text { on EM }\end{array}$ \\
\hline $38 \mathrm{~b}$ & M & 0.5 & U.S. & $\begin{array}{l}\text { Dd, hypotonia, sz, muscle weakness, ophthalmoparesis, } \\
\text { hypoventilation, abnl brain MRI, CIII and CIV deficiency, } \\
\text { lactic acidosis }\end{array}$ \\
\hline $39 \mathrm{bm}$ & F & 2.5 & U.S. & Dd, hypotonia, diarrhea, $\mathrm{ftt}$, organic aciduria, $\mathrm{CI}$ deficiency \\
\hline $40 \mathrm{~m}$ & $\mathrm{~F}$ & 1 & Dpln, $50 \%$ & $\begin{array}{l}\text { Dd, hypotonia, ataxia, peripheral neuropathy, fatigue, } \\
\text { cardiomyopathy, muscle weakness, rp, lactic acidosis, } \\
\text { elevated transaminases, increased subsarcolemmal } \\
\text { mitochondrial accumulation }\end{array}$ \\
\hline
\end{tabular}


Table 3

Continued

\begin{tabular}{|c|c|c|c|c|}
\hline Number & Sex & $\begin{array}{c}\text { Onset } \\
\text { age of SNHL (y) }\end{array}$ & Mutation \% heteroplasmy & $\begin{array}{c}\text { Clinical, metabolic, histochemical, and biochemical features }{ }^{b} \\
\text { in addition to hearing loss }\end{array}$ \\
\hline $41 \mathrm{~b}$ & $\mathrm{~F}$ & 5.0 & $\begin{array}{l}\text { T489C (mtTF binding) } \\
\text { G13708A (A458T) } \\
\text { LHON secondary }\end{array}$ & $\begin{array}{l}\text { Dd, autism, ataxia, sz, lactate peak on brain MRS, abnl brain } \\
\text { MRI }\end{array}$ \\
\hline $42 \mathrm{~b}$ & M & 8 & U.S. & $\begin{array}{l}\text { Dd, hypotonia, ataxia, fatigue, muscle weakness, ptosis, } \\
\text { peripheral neuropathy, GI dysmotility, cyclic vomiting, } \\
\text { lactic acidosis, low plasma carnitine }\end{array}$ \\
\hline $43 \mathrm{~m}$ & $\mathrm{~F}$ & 1 & U.S. & Dd, sz, hypotonia, abnl brain MRI, CIII deficiency \\
\hline $44 \mathrm{~b}$ & M & 0.5 & U.S. & Dd, hypotonia, weakness, hypoventilation \\
\hline $45 \mathrm{~b}$ & $\mathrm{~F}$ & 2 & U.S. & Dd, hypotonia, ataxia, leukodystrophy, muscle weakness \\
\hline
\end{tabular}

${ }^{a}$ Diagnostic criteria: all patients met definite criteria for mitochondrial disease.

${ }^{b}$ Sensorineural hearing loss (SNHL) is present in every patient.

Dd, developmental delay; CPEO, chronic progressive external ophthalmoplegia; C (I, II, III IV, V), Complex I, II, III, IV; dpln; depletion, \% of age-matched mean; dln, deletion, $\%$ of deletion mutant mtDNA molecules; amp, amplification of mitochondria copy number; abnl, abnormal; b, blood; m, muscle; $\mathrm{k}$, kidney; h, hair follicles; c, buccal mucosal cells; prolif, proliferation; cox, cytochrome c oxidase; rp, retinitis pigmentosa; Sz, seizures; EM, electron microscopy; MRS, magnetic resonance spectroscopy; GI dysmotility, gastrointestinal dysmotility; FTT, failure to thrive; US, unknown significance, including silent alterations and reported polymorphisms of either no significance or unknown significance.

point mutations or single deletions and within this group, $78 \%$ $(14 / 18)$ of the patients had mutations in mitochondrial tRNA genes.

\section{Patients with proven pathogenic mutations}

Eighteen out of 45 patients (40\%) in our cohort with hearing loss associated with mitochondrial cytopathy were found to have either single deletions or point mutations with proven pathogenicity. Within this group, the most common molecular mechanism was represented by mutations in mitochondrial tRNA genes observed in 15 patients $(83 \%)$ and the most frequent mutation was the $\mathrm{A} 3243 \mathrm{G}$ transition in the mitochondrial tRNA ${ }^{\text {Leu(UUR) }}$ gene which was found in 12 patients (66\% of the patients in group I) with the mutant heteroplasmy in blood varying from $10-66 \%$ (Table 3 ). The majority (7/12, $58 \%$ ) of patients carrying the A3243G mutation exhibited a step-wise, abrupt hearing loss in association with stroke-like episodes whereas the remainder $(5 / 12,42 \%)$ of the patients with no stroke-like episodes had a more gradual onset of SNHL. No correlation was found between mutant load in blood and hearing loss in decibels $(\mathrm{dB})$ and between degree of severity of hearing loss and the patient's age in this group of patients. The spectrum of clinical manifestations of the patients with MELAS ranged from milder features such as short stature, failure to thrive, hypotonia, and exercise intolerance to more severe ones such as pancreatitis, diabetes, cardiomyopathy, cardiac arrhythmias, seizures, and stroke-like episodes. The majority of patients $(9 / 12,75 \%)$ had isolated cochlear involvement whereas $1(1 / 12,8 \%)$ exhibited both cochlear and retrocochlear involvement and $2(2 / 12,17 \%)$ had isolated retrocochlear involvement.

Only one patient in our cohort (patient $14 \mathrm{~m}$ ) carried the common A8344G MERRF mutation (95\% mutant load on skeletal muscle specimen). This patient also carried a novel homoplasmic mutation T5553C in the mitochondrial tRNA ${ }^{\text {trp }}$ gene.

The novel $\mathrm{G}$ to $\mathrm{A}$ transition in position $5783 \mathrm{~A}$ was found in the stem region of the $\mathrm{T}$ arm of $\mathrm{RRNA}^{\text {cys }}$. This novel mutation, disrupting a base-pair in an evolutionarily highly conserved region, was present at $95 \%$ and $96 \%$ mutant heteroplasmy in the renal tissue and skeletal muscle, respectively (Table 3, I-18k, mt4618) with multi-systemic disease involving neuroophthalmological, cardiac, musculoskeletal, renal, gastrointestinal, and endocrine systems. Mitochondrial respiratory chain enzyme assays on skeletal muscle revealed deficiencies of complexes I, III, and IV and histopathology revealed abundant mitochondria with concentric cristae.

Patient $1 \mathrm{~b}$ was found to carry the A1555G mutation in the mitochondrial 12 seconds rRNA gene and in addition, she harbored multiple mtDNA deletions (Table 3). This patient exhibited multi-systemic CNS and musculoskeletal symptoms in addition to diabetes and hearing loss and a family history significant for maternally inherited hearing loss (MIHL).

The G14459A was the only mitochondrial protein coding gene mutation responsible for the disease phenotype found in this cohort of patients (patient $17 \mathrm{~m}$ ) (Table 3).

Two patients (15m and $16 \mathrm{~m}$ ) carried a single deletion of mitochondrial DNA. Patient $15 \mathrm{~m}$ had features of CPEO plus and patient $16 \mathrm{~m}$ had Kearns Sayre syndrome (Table 3).

TTGE analysis identified numerous polymorphisms (data not shown). However, most are silent substitutions leading to conserved amino acid changes or missense changes not located in structurally or functionally important regions without clinical consequences.

\section{Patients with multiple mtDNA deletions and alterations in mtDNA copy number}

Since there is variation in muscle mtDNA content among individuals, only a mtDNA content of $\leq 30 \%$ or $>140 \%$ of 
age-matched mean was considered significant. Twenty-four percent $(11 / 45)$ of the patients in the cohort exhibited alterations in mtDNA copy number including mtDNA depletion, over-replication, and multiple deletions (group II) with 5 patients (patients $19 \mathrm{~m}, 21 \mathrm{~m}, 25 \mathrm{~m}, 26 \mathrm{~m}$, and $28 \mathrm{~m}$ ) exhibiting mitochondrial DNA depletion, 4 patients (patients $20 \mathrm{~m}, 22 \mathrm{~m}$, $23 \mathrm{~m}$, and $29 \mathrm{~m}$ ) exhibiting mitochondrial DNA over-replication, one patient (patient $24 \mathrm{~m}$ ) with multiple mtDNA deletions, and one patient (patient $25 \mathrm{~m}$ ) that concomitantly had multiple mtDNA deletions and mtDNA depletion (Table 3 ).

\section{Patients with hearing loss and multi-systemic mitochondrial cytopathy and no significant mtDNA alterations}

The remainder of the patients $(16 / 45,36 \%)$ did not harbor any alterations in mtDNA (group III). Analysis of other mutations that have been associated with non-syndromic hearing loss identified one patient (patient 34b) with a T961G transversion and a patient (patient 33b) with a 961 delTinsC3 (C5TC4C12) in the mitochondrial 12S rRNA gene (Table 3).

\section{DISCUSSION}

Given the difficulty of studying RC defects in the absence of perfect diagnostic criteria or a gold standard test for the diagnosis of mitochondrial disease, the modified Walker criteria represent the best diagnostic tools currently available.

Sensorineural hearing loss is a commonly observed clinical feature in mitochondrial cytopathies. ${ }^{27}$ The percentage of sensorineural hearing loss caused by mtDNA mutations is not very well known. According to Hutchin and Cortopassi, ${ }^{2}$ as much as $15 \%$ of familial non-syndromic postlingual hearing loss shows a pattern of transmission compatible with maternal inheritance. However, it is not known to what extent mtDNA mutations are responsible for that observed transmission pattern and more studies are required to elucidate that hypothesis. The frequency of hearing loss in mitochondrial cytopathies has been found to be quite variable $(19.8-80 \%)$ based on different studies. ${ }^{7,28}$ Scaglia et al. ${ }^{29}$ found sensorineural hearing loss in $21 \%$ of subjects belonging to a cohort of patients with definite mitochondrial disease not carrying the $\mathrm{A} 1555 \mathrm{G}$ mutation. This was consistent with the results of a survey of review articles (appearing between 1984 and 1993) conducted by Gold and Rapin that showed $19.8 \%$ of the patients with mitochondrial cytopathies exhibiting SNHL.2,7 Our current study found a higher frequency (40\%) of SNHL in a population of patients with definite mitochondrial cytopathies. We assume that the results of studies conducted by Gold and Rapin and Scaglia et al. ${ }^{7,29}$ could represent an underestimate since not all patients underwent a formal hearing evaluation as part of their diagnostic work-up and the patients who did not undergo the evaluation with a presumably normal hearing history were assumed to have a normal hearing. In addition, the ascertainment criteria used in the study conducted by Gold and Rapin were not specified. ${ }^{7}$

In that same report, a total of 117 individual case reports of mitochondrial cytopathies were reviewed and progressive hearing loss was reported in the majority (59\%) of patients that were classified as having mitochondrial cytopathies. ${ }^{7}$ Edmonds et al. ${ }^{28}$ found that hearing loss was the most common clinical finding associated with mitochondrial disease and $80 \%$ of the patients undergoing testing had evidence of hearing loss or significant auditory dysfunction. This observed discrepancy could be due to different stringency in the diagnostic criteria compared to the modified diagnostic criteria ${ }^{18}$ used in our study. Another factor to consider could be the different age range of the patients. The median age of patients in our study was 7 years. However, SNHL was noted at a mean age of 25.2 years in the report by Gold and Rapin. ${ }^{7}$ It is possible that older patients may have a higher frequency of hearing loss due to their protracted clinical course. The median age of onset of SNHL in the paper by Edmonds et al. was not stated. ${ }^{28}$

More than one-third $(18 / 45,40 \%)$ of our patients harbor well known mtDNA mutations typically associated with classical mitochondrial syndromes such as MELAS, MERRF, CPEO plus and Kearns Sayre syndrome. This percentage is higher than the $11 \%$ and $16 \%$, respectively, reported by Scaglia et al. and Skladal et al., ${ }^{29,30}$ which included only pediatric patients. This discrepancy could be due to the inclusion of several adults in whom mtDNA mutations are more prevalent. ${ }^{31} \mathrm{An}$ other factor could be the more extensive mutational analysis performed in our selected group of patients.

Non-specific encephalomyopathy is another clinical presentation that accounts for a third of the cases in our patients with SNHL and associated mitochondrial disease. Most of the patients with these non-specific features are in a pediatric age range consistent with the findings reported by Scaglia et al. and Skladal et al. ${ }^{29,30}$

There was a much later age of onset of SNHL for the group with pathogenic mtDNA point mutations and single deletions when compared with the two other groups (Kruskal Wallis $P<$ 0.001). This statistically significant difference also applied when the age of onset of clinical features and age at diagnosis between the three groups where compared (Kruskal Wallis $P<$ 0.001 ). These findings would support the hypothesis that there are clinical differences in patients with mitochondrial cytopathies as a result of mtDNA compared with nuclear DNA mutations and greater clinical severity, earlier onset and a possible autosomal recessive inheritance in the latter. ${ }^{31}$

No correlation between the degree of hearing loss and the number and severity of clinical and neurological symptoms (hypo- or hypertonia, ataxia, movement disorder, ptosis, external ophthalmoplegia, frequency of seizures and stroke-like episodes, peripheral neuropathy, brainstem alterations, visual impairment, and mental retardation) could be found in our cohort, suggesting that the degree of hearing loss could not be used as a prognostic indicator of disease progression. It should be noted that this study was design to collect retrospective qualitative data rather than quantitative data.

This study demonstrates that cochlear damage is the predominant mechanism of SNHL in mitochondrial cytopathies. ${ }^{32,33}$ Otoacoustic emissions, a sensitive index of cochlear outer hair cell function, were universally absent in all patients with moderate or severe hearing loss. Since the energy molecule ATP is essential for 
the function of the stria vascularis and cochlea hair cells to maintain the ionic gradients required for sound signal transduction, ATP depletion would lead to the compromise of these postmitotic metabolically active tissues, resulting in SNHL. ${ }^{34}$

A minority of patients in our cohort who presented with mild hearing impairment exhibited normal otoacoustic emissions and abnormal ABERs suggesting that a retrocochlear dysfunction like that observed in auditory neuropathy may be present. Given the fact that these patients had normal imaging studies that did not suggest acoustic nerve damage, these patients could exhibit a progressive auditory neuropathy. Auditory neuropathy or dys-synchrony is a recently described pattern of hearing loss, diagnosed in association with audiological findings of abnormal brainstem responses in combination with normal otoacoustic responses. ${ }^{35}$ It has been reported in patients with mitochondrial cytopathies, more specifically in Leber hereditary optic neuropathy associated with the G11778A mutation. ${ }^{36}$ Further investigations are necessary to determine whether central auditory neuropathy is a more common feature of mitochondrial cytopathies.

Most of the point mutations were found in tRNA genes of mitochondrial genome alluding to a potential dysfunction of mitochondrial protein synthesis that could be deleterious to the cochlea. ${ }^{2}$

\section{Mutations in mitochondrial tRNA genes}

Sixty-seven percent $(12 / 18)$ of the subjects with mtDNA alterations carried the common A3243G mutation in the mitochondrial tRNA ${ }^{\mathrm{Leu}}$ gene. The frequency of this mutation in our cohort is higher than that reported by Chinnery et al. in a group of patients with hearing loss due to mtDNA defects. ${ }^{32}$ The high frequency of this mutation in patients with hearing loss and mitochondrial multi-systemic cytopathy is not surprising since it is the most commonly found mtDNA mutation. ${ }^{2} \mathrm{Sim}-$ ilarly, Kadowaki and coworkers reported this mutation in three out of five subjects with diabetes and deafness. ${ }^{37}$ In addition, almost three-fourths of patients with classic MELAS syndrome suffer from bilateral sensorineural hearing loss ${ }^{38}$; although other patients carrying the same mutation may only suffer from diabetes and/or deafness. In our cohort, all patients with this mutation had other features of mitochondrial disease. In the majority of the reported cases, diabetes usually starts before the onset of hearing loss. ${ }^{37,39}$ Of interest, however, most $(11 / 12,92 \%)$ of the patients with this mutation in the cohort exhibited SNHL with no concurrent evidence of glucose intolerance or diabetes.

Fifty-eight percent (7/12) of the patients with this mutation experienced stepwise, abrupt loss of hearing in association with stroke-like episodes. This is in agreement with the findings by Chinnery et al. where the majority of the patients (80\%) carrying the A3243G mutation exhibited an abrupt, stepwise hearing loss which usually occurred in association with strokelike episodes. ${ }^{32}$ Moreover, this was also observed by Sue et al. suggesting that acute metabolic compromise of the stria vascularis may cause irreversible functional loss of the cochlear hair cells. ${ }^{33}$
In our patients with the A3243G mutation, the correlation between mutation load in blood and their mean age-corrected hearing loss in decibels did not reach statistical significance and no relationship was observed between severity of hearing loss and patient's age. This was in agreement with the observations of Chinnery et al. ${ }^{32}$ We could speculate that a direct correlation between the percentage of mutant mtDNA in skeletal muscle and the mean age-corrected hearing loss in $\mathrm{dB}$ may exist however the diagnosis in our MELAS cohort was established mostly in blood.

Most of our patients with SNHL and a A3243G mutation had cochlear involvement. This finding seemed to be in agreement with a previous publication. ${ }^{33}$ One of our patients $(\mathrm{Pa}-$ tient 8) carrying this mutation presented with both cochlear and retrocochlear involvement and had mild hearing loss. It has been suspected that retrocochlear dysfunction is a secondary event to primary cochlear damage and that it would be seen with advanced disease..$^{40}$ However, the finding of retrocochlear involvement with mild hearing loss suggests that it is not always observed with advanced inner ear disease. In addition, concurrent cochlear and retrocochlear involvement in patients with the A3243G mutation MELAS syndrome was also observed by Zwirner and Wilichowski suggesting that retrocochlear dysfunction may also occur in this mitochondrial syndrome. ${ }^{41}$

A novel heteroplasmic G5783A mutation in the mitochondrial tRNA ${ }^{\text {cys }}$ gene was discovered. This mutation is undoubtedly pathogenic since it is heteroplasmic in the renal and skeletal muscle tissue of the patient and it is located in the highly conserved stem region that is important in the maintenance of structural integrity and stability of mitochondrial tRNAs. ${ }^{33-35}$ This patient presented with early onset deafness expanding the molecular spectrum of hearing loss associated with multsystemic mitochondrial.

\section{MtDNA single deletions}

Single mtDNA deletions may also lead to hearing loss. ${ }^{17}$ In our study, the clinical findings of SNHL in patients with single mtDNA deletions were very different to the ones observed in patients with mutations in mitochondrial tRNA genes. Both patients with single mtDNA deletions displayed a slowly progressive, bilateral, symmetrical, high frequency SNHL. This finding further supports what has been reported by Chinnery et al. ${ }^{32}$

\section{Missense mutations in mitochondrial protein-coding genes}

One of our patients in this cohort presenting with encephalopathy and mild sensorineural hearing loss exhibited a homoplasmic G14459A mutation in the ND6 gene. This mutation, which changes a conserved alanine to valine, has been found to be responsible for LHON, LHON plus dystonia, and pediatric dystonia in previously published cases. ${ }^{42,43}$ Our patient had sensorineural hearing loss with evidence of retrocochlear involvement. Trivial sensorineural hearing loss with an abnormal ABER has been previously reported in another patient carrying this mutation. ${ }^{42}$ In addition, sensorineural hearing loss with retrocochlear involvement has also been observed 
in two additional patients with LHON and the G11778A mtDNA mutation. ${ }^{36}$ These findings further support the notion that patients with primary LHON mutations should undergo a hearing evaluation as part of their diagnostic work-up and expands the spectrum of primary LHON mutations associated with SNHL.

Missense LHON secondary mutations, 15257GA (D171N in cyt b),15812GA (V356M in cyt b), and 4216TC (Y304H in ND1) were also found in patient $28 \mathrm{~m}$ whose skeletal muscle specimen contained reduced amount (25\% of age-matched mean) of mtDNA. Since the molecular defects responsible for the observed mtDNA depletion in this patient has not been identified, it is not clear if these secondary LHON mutations would have any effect on the clinical expression of hearing impairment.

\section{Mitochondrial ribosomal RNA gene mutations}

The 1555 AG mutation has been shown to be associated with aminoglycoside induced non-syndromic hearing loss due to the resemblance of the mutant $12 \mathrm{~S}$ rRNA to bacterial $16 \mathrm{~S}$ rRNA structure that binds to antibiotics. ${ }^{11,12}$ Variable penetrance of hearing loss and a mild biochemical defect associated with this mutation suggests that by itself it may not be sufficient to cause the phenotype however other factors such as exposure to aminoglycosides, nuclear modifier genes, and/or other mtDNA mutations may modify the penetrance of hearing loss. ${ }^{44,45}$ The patient carrying this mutation in our cohort exhibited multi-systemic involvement (ataxia, hypotonia, exercise intolerance, peripheral neuropathy, and diabetes) in addition to sensorineural hearing loss. Although mostly associated with aminoglycoside-induced and non-syndromic hearing loss, this mutation has been previously described in one family with Parkinson disease, one with spinal and pigmentary disturbances, and in one case of a woman with restrictive cardiomyopathy. ${ }^{46-48}$ However these diverse/heterogeneous manifestations might not be causally related to this mutation and may be expressed secondary to the presence of other genetic and/or environmental factors. In our patient, multiple mtDNA deletions were detected by PCR, however the significance of this finding is unclear since deletions of the mitochondrial genome accumulate in humans during the aging process. ${ }^{49}$

We identified one patient with the 961TG mutation and one patient with the 961 delTins $\mathrm{C}_{3}$ (nt956 to nt $965, \mathrm{C}_{5} \mathrm{TC}_{4}$ to $\mathrm{C}_{12}$ ) in the mitochondrial $12 \mathrm{~S}$ rRNA gene. However, the clinical significance of these mutations in hearing impairment is uncertain. The 961TG mutation was found in 3\% of pediatric patients with non-syndromic deafness. ${ }^{50,51}$ The $\mathrm{C}$ insertion at 961 was found to modify the phenotypic expression of deafness associated with 1555 AG. ${ }^{50,51}$ This mutation was not found in 226 Caucasian and 364 Chinese control subjects and it was suggested that this mutation might play a role in the pathogenesis of hearing loss. ${ }^{50,51}$ However Kobayashi et al. found that although $2 \%$ of patients with SNHL carried the 961delT mutation, a similar frequency was found in the general population and that hearing loss did not cosegregate with the presence of this mutation. ${ }^{52}$

\section{Alterations in mitochondrial copy number}

MtDNA depletion, over-replication, or multiple deletions may be secondary to a primary nuclear gene defect. Since the nuclear gene defect affects the mtDNA copy number or the integrity of the mtDNA, the clinical expression is expected to be consistent with a mitochondrial cytopathy.

For the first time, we found that mtDNA over-replication can be associated with SNHL. MtDNA over-replication has been associated with a 2 - to 4 -fold increase in the mtDNA copy number ${ }^{53,54}$ in patients with an atypical form of Kearns Sayre syndrome caused by mitochondrial DNA deletion and in patients with a hepatocerebral form of neonatal lactic acidosis. These disorders, characterized from a biochemical standpoint, are associated with an abnormally increased amount of mtDNA, which does not translate into normal mitochondrial enzyme activities since the patients with the hepatocerebral phenotype were found to have severe respiratory chain defects. ${ }^{53}$ Only one of the patients in our cohort (patient $23 \mathrm{~m}$ ) demonstrated hepatocerebral involvement whereas the other three displayed a neuromuscular phenotype in addition to SNHL, expanding the clinical spectrum described in association with this alteration of mtDNA copy number. Thus far the molecular etiology of mtDNA overreplication has not been resolved.

Five patients were found with predominant mtDNA depletion ( $\leq 30 \% \mathrm{mtDNA}$ content of age-matched mean) and SNHL. These patients presented with a predominant neuromuscular phenotype and two had cardiac involvement. Mitochondrial DNA depletion syndromes are caused by defects of mtDNA replication and maintenance of deoxynucleotide pools and are transmitted mainly in an autosomal recessive fashion..$^{55}$ In the published literature, deafness has been reported in a phenotype characterized by a late onset and slower progression in four children with multi-systemic involvement and mtDNA depletion. Three of these patients met diagnostic criteria for Kearns-Sayre syndrome which has not been typically associated with mitochondrial DNA depletion. ${ }^{56}$ The median age of onset of hearing loss in our patients with mtDNA depletion was thirteen years. Since the majority of the patients affected with mtDNA depletion syndromes usually have an earlier onset and more severe course, ${ }^{55}$ it is possible that many of the patients described in the literature have succumbed prior to any formal hearing evaluation.

The two patients with multiple deletions and SNHL presented at 2 and 15 years of age, respectively, with a phenotype consistent with ptosis, ophthalmoparesis, hypotonia, ataxia, and seizures. These cases were sporadic and given the early age of onset of symptoms an autosomal dominant mode of inheritance caused by mutations in the Twinkle, ANT1, POLG genes is unlikely. An autosomal recessive form of PEO and multiple deletions has also been described caused by mutations in the $P O L G$ gene however the onset of PEO in these patients is usually in the late teens. ${ }^{57}$ 
In conclusion, we provided a clinical and molecular characterization of hearing loss associated with multi-systemic mitochondrial dysfunction in a population of patients with definite mitochondrial disease by using the modified adult criteria. SNHL was found to be progressive and for the most part symmetrical and initially affecting higher frequencies. Our findings support the notion that most cases of mitochondrial SNHL have a cochlear basis however retro-cochlear disease may occur in the SNHL found in mitochondrial cytopathies. No correlation was found between the degrees of hearing loss and the number and severity of neurological manifestations. The spectrum of mtDNA abnormalities associated with sensorineural hearing loss continues to expand. Thus, in addition to screening for common mtDNA point mutations and single deletions, a systematic evaluation for low percentage of multiple deletions and mtDNA content for either depletion or over-replication by real time quantitative PCR is helpful. Screening the entire mitochondrial genome for unknown mutations using an effective mutation detection method such as TTGE may also be necessary although in this study only one novel mutation (the 5783 GA transition in the mitochondrial tRNA ${ }^{\text {cys }}$ gene) was identified as the primary cause of the disease. A prospective study will then be needed to better assess the natural history of sensorineural hearing loss in such patients to evaluate the frequency of central auditory impairment in carefully defined hearing loss associated multisystemic mitochondrial cytopathy.

\section{ACKNOWLEDGMENTS}

The authors would like to thank referring physicians and patients for the participation in this study. This study was partly supported by a grant from The Muscular Dystrophy Association to LJC Wong.

\section{References}

1. Gorlin RJ, Toriello HV, Cohen MM. Hereditary Hearing Loss and Its syndromes. Oxford: Oxford University Press; 1994.

2. Hutchin TP, Cortopassi GA. Mitochondrial defects and hearing loss. Cell Mol Life Sci 2000;57:1927-1937.

3. Bitner-Glindzicz M. Hereditary deafness and phenotyping in humans. Br Med Bull 2002;63:73-94.

4. Estivill X, Fortina P, Surrey S, Rabionet R, et al. Connexin-26 mutations in sporadic and inherited sensorineural deafness. Lancet 1998;351:394-398.

5. Lench N, Houseman M, Newton V, Van Camp G, et al. Connexin-26 mutations in sporadic non-syndromal sensorineural deafness. Lancet 1998;351:415.

6. Morell RJ, Kim HJ, Hood LJ, Goforth L, et al. Mutations in the connexin 26 gene (GJB2) among Ashkenazi Jews with non-syndromic recessive deafness. N Engl J Med 1998;339:1500-1505.

7. Gold M, Rapin I. Non-Mendelian mitochondrial inheritance as a cause of progressive genetic sensorineural hearing loss. Int J Pediatr Otorhinolaryngol 1994;30:91104.

8. Van Camp G, Smith RJ. Maternally inherited hearing impairment. Clin Genet 2000; 57:409-414.

9. Fischel-Ghodsian N. Mitochondrial genetics and hearing loss: the missing link between genotype and phenotype. Proc Soc Exp Biol Med 1998;218:1-6.

10. Noller HF. Ribosomal RNA and translation. Annu Rev Biochem 1991;60:191-227.

11. Harpur ES. The pharmacology of ototoxic drugs. Br J Audiol 1982;16:81-93.

12. Zhao H, Li R, Wang Q, Yan Q, et al. Maternally inherited aminoglycoside-induced and non-syndromic deafness is associated with the novel C1494T mutation in the mitochondrial 12 S rRNA gene in a large Chinese family. Am J Hum Genet 2004; 74: 139-152.

13. del Castillo FJ, Rodriguez-Ballesteros M, Martin Y, Arellano B, et al. Heteroplasmy for the $1555 \mathrm{~A}>\mathrm{G}$ mutation in the mitochondrial $12 \mathrm{~S}$ rRNA gene in six Spanish families with non-syndromic hearing loss. J Med Genet 2003;40:632-636.
14. Bacino C, Prezant TR, Bu X, Fournier P, et al. Susceptibility mutations in the mitochondrial small ribosomal RNA gene in aminoglycoside induced deafness. Pharmacogenetics 1995;5:165-172.

15. Guan MX, Enriquez JA, Fischel-Ghodsian N, Puranam RS, et al. The deafness-associated mitochondrial DNA mutation at position 7445 , which affects tRNASer(UCN) precursor processing, has long-range effects on NADH dehydrogenase subunit ND6 gene expression. Mol Cell Biol 1998;18:5868-5879.

16. Ballinger SW, Shoffner JM, Gebhart S, Koontz DA, et al. Mitochondrial diabetes revisited. Nat Genet 1994;7:458-459.

17. Ballinger SW, Shoffner JM, Hedaya EV, Trounce I, et al. Maternally transmitted diabetes and deafness associated with a $10.4 \mathrm{~kb}$ mitochondrial DNA deletion. Nat Genet 1992;1:11-15.

18. Bernier FP, Boneh A, Dennett X, Chow CW, et al. Diagnostic criteria for respiratory chain disorders in adults and children. Neurology 2002;59:1406-1411.

19. Lahiri DK, Nurnberger JI. A rapid non-enzymatic method for the preparation of HMW DNA from blood for RFLP studies. Nucleic Acids Res 1991;19:5444.

20. Wong L-JC, Lam C. Alternative, noninvasive tissues for quantitative screening of mutant mitochondrial DNA. Clin Chem 1997;43:1241-1243.

21. Wong L-JC, Senadheera D. Direct detection of multiple point mutations in mitochondrial DNA. Clin Chem 1997;43:1857-1861.

22. Liang MH, Wong LJ. Yield of mtDNA mutation analysis in 2,000 patients. Am JMed Genet 1998;77:395-400.

23. Wong L-JC, Liang M-H, Kwon, H, Park J, et al. Comprehensive scanning of the whole mitochondrial genome for mutations. Clin Chem 2002;48:1901-1912.

24. Wong L-JC, Perng CL, Hsu CH, Bai RK, et al. Compensatory amplification of mitochondrial DNA in a patient with a novel deletion/duplication and high mutant load. J Med Genet 2003;40:E125.

25. Bai RK, Perng CL, Hsu CH, Wong LJ. Quantitative PCR analysis of mitochondrial DNA content in patients with mitochondrial disease. Ann N Y Acad Sci 2004;1011: 304-349.

26. Bai RK, Wong LJ. Simultaneous detection and quantification of mitochondrial DNA deletion(s), depletion, and over-replication in patients with mitochondrial disease. J Mol Diagn 2005;7:613-622.

27. Fischel-Ghodsian N. Mitochondrial deafness. Ear Hear 2003;24:303-313.

28. Edmonds JL, Kirse DJ, Kearns D, Deutsch R, et al. The otolaryngological manifestations of mitochondrial disease and the risk of neurodegeneration with infection. Arch Otolaryngol Head Neck Surg 2002;128:355-362.

29. Scaglia F, Towbin JA, Craigen WJ, Belmont JW, et al. Clinical spectrum, morbidity, and mortality in 113 pediatric patients with mitochondrial disease. Pediatrics 2004 114:925-931.

30. Skladal D, Sudmeier C, Konstantopoulou V, Stockler-Ipsiroglu S, et al. The clinical spectrum of mitochondrial disease in 75 pediatric patients. Clin Pediatr (Phila) 2003;42:703-710.

31. Rubio-Gozalbo ME, Dijkman KP, van den Heuvel LP, Sengers RC, et al. Clinical differences in patients with mitochondriocytopathies due to nuclear versus mitochondrial DNA mutations. Hum Mutat 2000;15:522-532.

32. Chinnery PF, Elliott C, Green GR, Rees A, et al. The spectrum of hearing loss due to mitochondrial DNA defects. Brain 2000;123:82-92.

33. Sue CM, Lipsett LJ, Crimmins DS, Tsang CS, et al. Cochlear origin of hearing loss in MELAS syndrome. Ann Neurol 1998;43:350-359.

34. Dallos P, Evans BN. High-frequency motility of outer hair cells and the cochlear amplifier. Science 1995;267:2006-2009.

35. Starr A, Picton TW, Sininger Y, Hood LJ, et al. Auditory neuropathy. Brain 1996 119:741-753.

36. Ceranic B, Luxon LM. Progressive auditory neuropathy in patients with Leber's hereditary optic neuropathy. J Neurol Neurosurg Psychiatry 2004;75:626-630.

37. Kadowaki T, Kadowaki H, Mori Y, Tobe K, et al. A subtype of diabetes mellitus associated with a mutation of mitochondrial DNA. N Engl J Med 1994;330:962-968.

38. Hirano M, Pavlakis SG. Mitochondrial myopathy, encephalopathy, lactic acidosis, and strokelike episodes (MELAS): current concepts. J Child Neurol 1994;9:4-13.

39. Olsson C, Zethelius B, Lagerstrom-Fermer M, Asplund J, et al. Level of heteroplasmy for the mitochondrial mutation A3243G correlates with age at onset of diabetes and deafness. Hum Mutat 1998;12:52-58.

40. Elverland $\mathrm{HH}$, Torbergsen T. Audiologic findings in a family with mitochondrial disorder. Am J Otol Nov 1991;12:459-465.

41. Zwirner P, Wilichowski E. Progressive sensorineural hearing loss in children with mitochondrial encephalomyopathies. Laryngoscope 2001;111:515-521.

42. Tarnopolsky MA, Baker SK, Myint T, Maxner CE, et al. Clinical variability in maternally inherited leber hereditary optic neuropathy with the G14459A mutation. Am J Med Genet 2004;124:A372-A376.

43. Gropman A, Chen TJ, Perng CL, Krasnewich D, et al. Variable clinical manifestation of homoplasmic G14459A mitochondrial DNA mutation. Am J Med Genet 2004; 124:A377-A382. 


\section{Scaglia et al.}

44. Bykhovskaya Y, Estivill X, Taylor K, Hang T, et al. Candidate locus for a nuclear modifier gene for maternally inherited deafness. Am J Hum Genet 2000;66:19051910.

45. Li X, Li R, Lin X, Guan MX. Isolation and characterization of the putative nuclear modifier gene MTO1 involved in the pathogenesis of deafness-associated mitochondrial 12 S rRNA A1555G mutation. J Biol Chem 2002;277:27256-27264.

46. Nye JS, Hayes EA, Amendola M, Vaughn D, et al. Myelocystocele-cloacal exstrophy in a pedigree with a mitochondrial $12 \mathrm{~S}$ rRNA mutation, aminoglycoside-induced deafness, pigmentary disturbances, and spinal anomalies. Teratology 2000;61:165-171.

47. Santorelli FM, Tanji K, Manta P, Casali C, et al. Maternally inherited cardiomyopathy: an atypical presentation of the mtDNA $12 \mathrm{~S}$ rRNA gene A1555G mutation. Am J Hum Genet 1999;64:295-300.

48. Shoffner JM. Oxidative phosphorylation disease diagnosis. Semin Neurol 1999;19: 341-351.

49. Cortopassi GA, Wong A. Mitochondria in organismal aging and degeneration. Biochim Biophys Acta 1999;1410:183-193.

50. Li R, Greinwald JH, Yang L, Choo DI, et al. Molecular analysis of the mitochondria $12 \mathrm{~S}$ rRNA and tRNASer(UCN) genes in paediatric subjects with non-syndromic hearing loss. J Med Genet 2004;41:615-620.
51. Li R, Xing G, Yan M, Cao X, et al. Cosegregation of C-insertion at position 961 with the A1555G mutation of the mitochondrial 12S rRNA gene in a large Chinese family with maternally inherited hearing loss. Am J Med Genet 2004;124:A113A117.

52. Kobayashi K, Oguchi T, Asamura K, Miyagawa M, et al. Genetic features, clinical phenotypes, and prevalence of sensorineural hearing loss associated with the 961delT mitochondrial mutation. Auris Nasus Larynx 2005;32:119-124.

53. Chabi B, Mousson de Camaret B, Duborjal H, Issartel JP, et al. Quantification of mitochondrial DNA deletion, depletion, and over-replication: application to diagnosis. Clin Chem 2003;49:1309-1317.

54. Wong L-JC, Perng C-L, Hsu C-H, Bai RK, et al. Compensatory amplificationof mtDNA in a patient with a novel deletion/duplication and high mutant load. JMed Genet 2003;40:E125.

55. Elpeleg O. Inherited mitochondrial DNA depletion. Pediatr Res 2003;54:153-159.

56. Barthelemy C, Ogier de Baulny H, Diaz J, Cheval MA, et al. Late-onset mitochondrial DNA depletion: DNA copy number, multiple deletions, and compensation. Ann Neurol 2001;49:607-617.

57. Spinazzola A, Zeviani M. Disorders of nuclear-mitochondrial intergenomic signaling. Gene 2005;354:162-168. 